\title{
La formación del profesorado en la RIEMS. Un estudio de caso en la Escuela Preparatoria 9 del SEMS de la Universidad de Guadalajara, ${ }^{1,2}$
}

\author{
Jon Olaskoaga-Larrauri, Carlos Mendoza-Sepúlveda y Elia Marúm-Espinosa
}

\begin{abstract}
RESUMEN
Este artículo propone una valoración de la formación recibida por el profesorado de la educación media superior con motivo de la introducción de la Reforma Integral de la Educación Media Superior (RIEMS) y del enfoque por competencias en este nivel educativo. La evaluación se ha realizado a partir de las opiniones del profesorado. Se ha preguntado al profesorado si la ayuda recibida por las autoridades académicas ha sido suficiente en lo que respecta a su formación en competencias y prácticas RIEMS; pero también si considera poseer dichas competencias y aplicar adecuadamente estas prácticas en el aula. El trabajo concluye que las opiniones de los docentes sobre la eficacia de las acciones formativas es bastante favorable, y que la capacitación del profesorado sigue siendo un instrumento esencial en la implementación de la reforma, entre otras cosas por su capacidad para socializar a la planta docente en los principios de la enseñanza por competencias.
\end{abstract}

Palabras clave: Reforma Integral de la Educación Media Superior (RIEMS), México, competencias docentes, prácticas docentes, profesorado.

Jon Olaskoaga-Larrauri

jon.olaskoaga@ehu.es Español. Doctor en Economía por la Universidad del País Vasco/Euskal Herriko Unibertsitatea (UPV/EHU), profesor Titular de Universidad de dicha Universidad. Temas de investigación diseño de políticas públicas, políticas sociales y gestión de la calidad en las instituciones universitarias.

Carlos Mendoza Sepúlveda

cm101550@gmail.com Mexicano. Doctor en Gestión de la Educación Superior por la Universidad de Guadalajara, México. Temas de investigación: calidad de la educación universitaria, gestión y liderazgo en las instituciones educativas.

\section{Elia Marúm Espinosa}

eliamarume@yahoo.com.mx Mexicana. Doctora en Economía, Universidad Nacional Autónoma de México (UNAM). Directora y profesora del Centro para la Calidad e Innovación de la Educación Superior del Centro Universitario de Ciencias Económico Administrativas de la Universidad de Guadalajara. Coordinadora de la Cátedra UNESCO "Género, liderazgo y equidad". Temas de investigación: gestión de la calidad de la educación superior, enfoque de género en la gestión y el presupuesto públicos, innovación educativa y la gestión del conocimiento y el emprendimiento social y la innovación educativa.

\footnotetext{
${ }^{1}$ Este trabajo se ha beneficiado de la financiación otorgada por la Universidad del País Vasco/Euskal Herriko Unibertsitatea (UPV/EHU) al grupo de investigación ECUALE, a través de la convocatoria de grupos de investigación. Referencia GIU: 13/42.

${ }^{2}$ Los autores desean manifestar su agradecimiento a los tres evaluadores anónimos que dictaminaron un borrador anterior de este artículo por sus atinados comentarios. Cualquier error que subsista en la versión definitiva del artículo es responsabilidad exclusiva de los autores.
} 


\section{A formação do professorado na RIEMS. Um estudo de caso na Escuela Preparatoria 9 do SEMS da Universidad de Guadalajara}

\section{RESUMO}

Este artigo propõe uma avaliação da formação recebida pelo professorado da educação média-superior com motivo da introdução da Reforma Integral da educação média-superior (RIEMS) e do enfoque por atribuições neste nível educativo. A avaliação se realizou a partir das opiniões do professorado. Foi perguntado ao professorado se a ajuda recebida pelas autoridades acadêmicas tem sido suficiente no que diz respeito a sua formação em atribuições e práticas RIEMS; mas também se considera a posse destas atribuições e a aplicação adequada destas práticas na sala de aula. O trabalho conclui que as opiniões dos docentes sobre a eficácia das ações formativas são bastante favoráveis, e que a capacitação do professorado continua sendo um instrumento essencial na implementação da reforma, entre outras coisas por sua capacidade para socializar a projeção docente nos princípios do ensino por atribuições.

Palavras chave: Reforma Integral da Educação Média-Superior (RIEMS), atribuições docentes, práticas docentes, professorado, México.

\section{Teacher training within the framework of the Comprehensive Reform of Higher Secondary Education. A case study in the High School 9 of the University of Guadalajara's Higher Secondary Education System}

\section{ABSTRACT}

This article proposes an assessment of the training received by teachers in higher secondary education when they put into practice the Comprehensive Reform of Higher Secondary Education (Reforma Integral de la Educación Media Superior, RIEMS) and the competency-based approach at this educational level. This assessment has been carried out on the basis of the opinions expressed by the teaching staff. Teachers have been asked whether the support received from the academic authorities has been sufficient in terms of their training in RIEMS skills and practices; but also whether they consider that they possess these skills and apply these practices adequately within the classroom. This article concludes that teachers' opinions on the effectiveness of training actions are quite favorable, and that teacher training keep being an essential instrument in the implementation of the reform, among other things because of its capacity to share with the teaching staff the principles of competency-based teaching.

Key words: Comprehensive reform of higher secondary education (RIEMS), teaching skills, teaching practices, teaching staff, Mexico.

Recepción: 13/12/16. Aprobación: 22/03/18. 


\section{Introducción}

En el ciclo escolar 2008-2009 entró en vigor en México la Reforma Integral de la Educación Media Superior (RIEMS), una ambiciosa actualización del nivel de la Educación Media Superior (EMS) que se planteaba con dos grupos de objetivos discernibles, aunque relacionados.

Por un lado, la RIEMS tenía la pretensión de reestructurar la EMS, hasta la fecha compuesta por subsistemas insuficientemente integrados. La falta de integración tenía serios efectos; por ejemplo, el escaso reconocimiento de algunos de los subsistemas; y, particularmente, las dificultades de los estudiantes para transitar entre subsistemas. Esta situación resultaba inadmisible, especialmente por comparación con otros ámbitos geográficos, como el europeo, donde el reconocimiento recíproco de los títulos y la portabilidad de los haberes académicos se ha logrado incluso en el ámbito internacional y en el nivel de la Educación Superior (Beerkens, 2008). En esta faceta de los objetivos de la RIEMS juega un papel determinante la certificación de los profesores y la incorporación de las escuelas al Sistema Nacional de Bachillerato (SNB), como mecanismos que generan credibilidad tanto hacia dentro como hacia fuera del sistema.

Por otro lado, la RIEMS también pretendía realizar avances en la calidad y la pertinencia de la educación que recibe el estudiantado en este nivel educativo. La RIEMS propone una modificación radical del enfoque de la enseñanza: se pretende que las actividades docentes se vuelquen hacia la consecución de determinadas competencias por parte del alumnado y que la propia enseñanza y sus resultados se definan, se planifiquen, se organicen y se evalúen en función del logro de dichas competencias.

La ambición de los objetivos suele ser pareja a la dificultad de su logro. Algunos analistas señalaron que los objetivos de la RIEMS serían inalcanzables teniendo en cuenta las circunstancias actuales de determinados elementos del sistema (González et al., 2015). Algunos argumentos se basaron en el profesorado y, en particular, en su formación y sus actitudes (Sosa y Ribeiro, 2014; Ibarra et al., 2012; López, 2011; Fonseca, 2011; Scheel, 2009). Se desconfiaba de que el profesorado estuviese suficientemente preparado para afrontar las novedades que representa el enfoque de la enseñanza por competencias, y se temía que los cambios solicitados en su trabajo fueran tan radicales que terminaran por poner al profesorado en contra de la reforma, una resistencia (Díaz-Barriga, 2010) que a su vez podría convertirse en el principal obstáculo para las intenciones reformistas (Macías, 2009), tal y como ha sucedido con ocasión de otras reformas en diferentes niveles educativos (Olaskoaga-Larrauri et al., 2015).

Se espera que la formación del profesorado tenga dos efectos: por un lado, la capacitación del profesorado en cuanto a la adquisición de determinadas competencias (establecidas en el acuerdo 447 de la Secretaría de Educación Pública [SEP], 2008) y la efectiva y adecuada ejecución de determinadas prácticas docentes; por otro, su socialización con los valores de la RIEMS, y particularmente con el enfoque del aprendizaje por competencias. La literatura ha subrayado el papel del cambio de la cultura profesional del profesorado como uno de los factores de éxito en las reformas educativas (Day, 2002; Van der Berg, 2002; Imbernón, 1994), en particular cuando dichas reformas no suponen simplemente la aplicación de nuevas técnicas de enseñanza en el aula, sino la implicación del profesor en la labor de diseñar y ensayar nuevos métodos adaptados a sus condicionamientos "locales"; en suma, cuando las reformas consisten en una constante "reinvención" de la tarea del profesor (Schmidt y Datnow, 2005; Little, 1993).

Para Lozano (2012), uno de los problemas a los que se enfrenta la RIEMS es la ausencia de una política de implementación de la reforma. Lo mismo sucede con Programa de Formación Docente de Educación media Superior (PROFORDEMS), cuyo éxito depende de la voluntad de las instituciones que ofrecen estudios de EMS, del interés que demuestre el 
profesorado en participar de los cursos formativos y de la disponibilidad de recursos financieros y humanos. En particular, alerta Lozano (2012), la voluntad institucional a veces se queda en el discurso y no se traduce en el apoyo institucional que el profesorado necesita para tomar parte en el programa, ni en la puesta en marcha de mecanismos de seguimiento de sus avances.

Este artículo tiene dos objetivos. El primero consiste en evaluar la formación que, con el objeto de facilitar la transición al enfoque por competencias, ha recibido el profesorado de la EMS en México. Este primer objetivo se desdobla en dos: por un lado se lleva a cabo un repaso general y crítico de PROFORDEMS, que se efectúa mediante fuentes secundarias. En segundo lugar se describen las percepciones que han desarrollado los propios docentes sobre la formación recibida. Este segundo enfoque requiere consultar a los docentes, pero permite una evaluación detallada de tres aspectos diferentes: la valoración de los docentes en torno a la influencia de las competencias y prácticas docentes RIEMS en la calidad de la enseñanza impartida en el nivel de la EMS; su percepción del apoyo de las autoridades académicas a la formación del profesorado; y su opinión sobre si las prácticas docentes de RIEMS se están aplicando efectivamente.

El segundo objetivo consiste en comprobar si la participación en PROFRODEMS ha tenido algún efecto en las percepciones de los docentes sobre los tres aspectos descritos.

$\mathrm{El}$ artículo se ordena en siete apartados. En el segundo apartado se resumen algunas características de PROFRORDEMS y se propone una primera revisión crítica de su evolución. En el tercer apartado se describe la Escuela Preparatoria en la que se llevó a cabo el estudio de caso en que se basa el artículo, así como los instrumentos empleados en la recolección de datos y las herramientas utilizadas en el análisis. El cuarto apartado comienza con el análisis de resultados, describiendo las opiniones del profesorado consultado a propósito de las competencias docentes auspiciadas por la RIEMS. El quinto hace lo mismo con las prácticas docentes que la RIEMS promueve. El sexto apartado valora la medida en que las respuestas del personal académico sobre todos estos asuntos dependen de su participación en PROFORDEMS. Finalmente, en el séptimo apartado se exponen algunas conclusiones.

\section{PROFORDEMS y formación del personal docente en competencias}

El Programa de Formación Docente de Educación Media Superior (PROFORDEMS) constituye uno de los instrumentos básicos de la RIEMS. Nació para asegurar que los docentes dispusieran de las competencias y llevaran a cabo las actividades sin las cuales la enseñanza por competencias que augura la RIEMS no podría darse.

El enfoque formativo que se adoptó con PROFORDEMS supuso al menos dos novedades con respecto al modo en que, en el pasado, se había afrontado la capacitación profesional del profesorado en México. En primer lugar se evitó el esquema habitual de formación "en cascada" (cf. Fonseca, 2011: 51), por el cual el docente que participa en un curso de formación transmite después lo recibido a sus colegas. En segundo lugar, la RIEMS involucra a la educación superior en la formación, acabando con una tradicional ausencia en la formación de los profesionales de este nivel (Alcántara y Zorrilla, 2010: 54). Originalmente la oferta formativa de PROFORDEMS consistía en un diplomado coordinado por la Asociación Nacional de Universidades e Instituciones de Educación Superior (ANUIES), y en algunas especialidades diseñadas e impartidas por la Universidad Pedagógica Nacional (UPN) (Lozano, 2015); pero con el tiempo se amplió la cooperación con un mayor número de universidades que diseñaron y pusieron en marcha programas adaptados a los lineamientos del acuerdo 447 de la SEP. Al mismo tiempo la UPN continuaba impartiendo cursos en línea. 
PROFORDEMS ha resultado, a juicio de varios analistas, insuficiente. Lozano ha destacado la magnitud del objetivo de formar a los miles de individuos que componen el profesorado de la EMS, más aun teniendo en cuenta la diversidad de las instituciones en las que trabajan (Villa, 2012) y su dispersión geográfica (371 657 docentes en diciembre de 2014, trabajando en 16457 escuelas; según recoge Lozano, 2015: 114). El primer objetivo para PROFORDEMS lo marcaba el propio acuerdo 447, que en su disposición transitoria cuarta prescribía que el profesorado de los planteles dependientes de la SEP y de sus órganos desconcentrados debería contar con las competencias descritas en el acuerdo al inicio del ciclo escolar 2009-2010; sin embargo, la realidad se impuso a los deseos de la SEP y, en lo que podría definirse como el primer reporte de la Subsecretaría de Educación Media Superior (SEMS) sobre los avances de PROFORDEMS (SEP-SEMS, 2009), se reconoció que 2000 maestras y maestros de bachillerato se habían acreditado hasta 2009, dentro una población de algo más de 51000 individuos (Lozano, 2015: 120).

La valoración que la Auditoría Superior de la Federación (ASF) realizó en 2011 de los logros de PROFORDEMS tampoco fue muy favorable (ASF, 2011). Su informe describe carencias en la forma de medir resultados y realizar el puntual seguimiento que un programa de estas características exige. Según la Auditoría, no se formularon indicadores ni metas de cobertura relativas a la atención del profesorado. La falta de planificación se conjuga con la falta de transparencia y publicidad de los resultados, de la que se quejan también otros analistas (Lozano, 2015). El informe también resalta el escaso número de profesores que habían logrado su certificación y refiere, en su punto octavo, que en 2011 la SEP solamente había cumplido un $84.4 \%$ con el objetivo de actualizar a todos los profesores de las escuelas públicas federales, objetivo que se había planteado, como ya se ha dicho, para el comienzo del ciclo 2009-2010.

El 3 de septiembre de 2013, se celebró en
Monterrey la XLIV Reunión Ordinaria del Consejo Nacional de Autoridades Educativas, en la que se presentó un documento (SEP-SEMS, 2013) donde se facilitaban algunas cifras relativas a la formación del profesorado en PROFORDEMS. Según estas cifras, de un total de 180852 profesores (que no incluyen a algunas instituciones importantes como la Universidad Nacional Autónoma de México (UNAM), que no quiso incorporarse a la RIEMS), 112334 se habían inscrito en PROFORDEMS, 75343 habían egresado de los cursos, y solamente 13764 (un 7.6\%) habían logrado su certificación.

Los informes de labores de la SEP no aportan mucha más información sobre la medida de los logros de PROFORDEMS. El segundo informe de la SEP para el curso 2013-2014 (SEP, 2014: 63) informa de una cobertura formativa del $56.2 \%$ de los docentes que trabajaban en los planteles dependientes de instituciones públicas pero, como señala Lozano (2015), no se especifica si el dato se refiere a docentes formados o a docentes certificados. Este mismo autor concluye que en PROFORDEMS ni hay capacidad para atender a la población total, ni existen mecanismos para garantizar la permanencia de los docentes en el programa, ni menos aun para asegurar que se alcanza la meta final de la certificación.

\section{Estudio de caso en la Universidad de Guadalajara}

El presente artículo propone una valoración de los efectos de la formación recibida por el profesorado de la EMS, y en particular del programa PROFORDEMS, a partir de un estudio de caso realizado en la Escuela Preparatoria 9, del Sistema de Educación Media Superior de la Universidad de Guadalajara (SEMS de la UdeG).

Aunque cuestionado por algunos autores, el método conocido como estudio de caso ha prosperado como metodología científica en áreas científicas como la educación, o la investigación social en general (Martínez, 2006). Uno de los rasgos que mejor 
caracteriza a los estudios de caso consiste en que con esta metodología los fenómenos se estudian en sus contextos reales y concretos (Villareal y Landeta, 2010) lo cual permite al investigador reconocer, al menos en parte, la múltiple causalidad que opera sobre ellos. Para enfrentarse a esta causalidad múltiple el estudio de caso precisa disponer de fuentes de información diversas; de ahí que en él se conjuguen fuentes de evidencia cuantitativas y cualitativas, y se empleen diferentes técnicas de investigación, desde la revisión documental, hasta las encuestas o las entrevistas en profundidad a informantes clave.

Consecuentemente, en el estudio de caso la contextualización del caso de estudio constituye un requisito metodológico, más que un mero ejercicio de cortesía para con el lector; requisito que se aborda en las siguiente líneas.

Este artículo se basa en un estudio de caso realizado en la Escuela Preparatoria 9 del SEMS de la UdeG. La escuela inició sus actividades en 1991 en el sur del municipio de Zapopan, en la conurbación de Guadalajara, y en 2008 se amplió con la incorporación de un módulo sito en el municipio de Tlaquepaque, colindante con Guadalajara. El plantel ofrece un plan de estudios de bachillerato general por competencias con modalidad escolarizada y opción educativa presencial.

En 2014, fecha en la que se realizó el estudio, la escuela contaba con 184 profesores y prestaba sus servicios a un total de 4093 alumnos, de los cuales 2379 eran mujeres. Empleando como referencia al SEMS, el tamaño de esta preparatoria puede describirse como mediano. El cuestionario se entregó a toda la planta docente y se obtuvieron 176 respuestas, los ocho cuestionarios restantes no fueron completados por hallarse sus destinatarios en situaciones de baja o licencia laboral.

En lo que afecta al objetivo de este artículo, debe tenerse en cuenta que la Escuela Preparatoria 9 abordó de manera muy temprana la puesta en marcha de una enseñanza por competencias. Las acciones institucionales se iniciaron en 2003 con una amplia participación de las instancias académicas, directivas y de los órganos de gobierno del sistema. En 2006 se redactó un documento denominado "Bachillerato General por Competencias" (Haro del Real et al., 2007) que fue difundido a partir de junio de 2007 a través de las direcciones y coordinaciones académicas de las escuelas. También se llevó a cabo un importante esfuerzo de recolección de información que condujo al "Primer Censo de Necesidades de Formación Docente" (SEMS de la UdeG, 2008).

La oferta formativa en el SEMS se concretó en una maestría en Tecnologías para el Aprendizaje, un diplomado en Desarrollo de Habilidades del Razonamiento Lógico y Matemático, y un diplomado en Habilidades Docentes para el Siglo XXI, que lograron una participación aproximada de 3000 académicos y autoridades del nivel (SEMS de la UdG, 2009). En 2008, cuando se puso en marcha la RIEMS, el SEMS de la UdeG logró reportar 1380 profesores preparados con herramientas metodológicas adecuadas a una didáctica constructivista y el desarrollo de competencias en sus alumnos, así como con actitudes favorables hacia el nuevo tipo de enseñanza (SEMS de la UdeG, 2008); y ya con la RIEMS en vigor, en 2009, se puso en marcha un nuevo paquete de medidas formativas más directamente orientadas a las competencias docentes del acuerdo 447 (SEMS de la UdG, 2009).

El programa de formación diseñado para el SEMS de la UdeG se complementó con actuaciones específicas en algunas de las escuelas. En la Preparatoria 9 se llevaron a cabo en 2009 la III y la IV Semanas del Trabajo Colegiado, con la asistencia del 95\% de los docentes; también se puso en marcha un proceso denominado "Talleres de Microplaneación", orientado a la planificación de los cursos por competencias, para los docentes de cada una de las unidades de aprendizaje. La formación se completó con otros cursos, algunos de ellos específicamente diseñados para la adquisición de competencias docentes. El 
informe de actividades del SEMS de la UdeG (2009) aporta indicadores de asistencia a los cursos siempre por encima del $75 \%$ y trayectoria ascendente desde el momento de la entrada en vigor de la RIEMS.

Por otro lado, la Escuela 9 se fijó desde un principio los objetivos de llevar a cabo una transición eficaz hacia el Bachillerato por Competencias (BGC) incorporarse al Sistema Nacional de Bachillerato. En 2012 organizó, con este objeto, un Comité de Apoyo Técnico para la Transición al BGG, con el mandato de elaborar un diagnóstico de la situación. Desde entonces la Escuela ha realizado gestiones para apoyar a sus académicos en la obtención de sus diplomados PROFORDEMS y en el logro de su certificación. Todas estas gestiones han derivado en un notable éxito de la Escuela en lo que se refiere a su incorporación al Sistema Nacional de Bachillerato. La Escuela entró en el sistema en 2012 (en su nivel III). Posteriormente la Escuela ha sido evaluada en 2013 y en 2016, alcanzando en esta última evaluación el nivel I del SNB.

Es en el contexto descrito en los últimos párrafos en el que deben interpretarse las opiniones de los profesores sobre la formación que han recibido para transitar hacia la enseñanza por competencias. Estas opiniones se obtuvieron mediante una encuesta que se pasó en el mes de octubre de 2014. El cuestionario se diseñó con la intención de conocer varios aspectos relacionados con la RIEMS. En dos apartados del cuestionario se recogieron preguntas relativas a las competencias de la RIEMS, de acuerdo con el acuerdo 447 de la SEP (SEP, 2008) y a una serie de prácticas docentes que se derivan de los atributos en los que se concretan dichas competencias. Se solicitó al profesorado que declarara su grado de acuerdo o desacuerdo (en escalas de Likert con cinco categorías de respuesta) con la importancia de cada competencia y práctica docente desde el punto de vista de la calidad educativa; con la afirmación de que el apoyo recibido por las autoridades es suficiente para su formación en cada una de ellas; y con la afirmación de que las posee o aplica. Finalmente, el cuestionario recoge una serie de variables de identificación, incluyendo información sobre la participación del profesorado en PROFORDEMS.

El análisis de las respuestas obtenidas que se presenta en este artículo se lleva a cabo mediante estadísticos descriptivos, principalmente frecuencias relativas de respuesta y contrastes Chi cuadrado $(\chi 2)$. La prueba $\chi^{2}$ es una prueba no paramétrica de independencia que permite identificar si dos variables cualitativas están relacionadas o no. En el caso particular de este artículo se trabaja con dos variables dicotómicas, es decir, que admiten solamente dos categorías. La primera señala si un determinado individuo está de acuerdo o no con una afirmación (en el artículo la prueba se aplica sobre múltiples afirmaciones) y la segunda indica si el individuo dispone o no de PROFORDEMS. Cuando la prueba resulta significativa se descarta la independencia entre las dos variables y, por tanto, debe admitirse que los individuos con PROFORDEMS muestran una propensión diferente (mayor o menor, según el caso) a estar de acuerdo con la correspondiente afirmación.

\section{Competencias RIEMS: importancia percibida y formación recibida}

$\mathrm{El}$ aspecto más relevante en la evaluación de un programa formativo debería ser el de su pertinencia, especialmente cuando la formación está destinada a desarrollar o a consolidar las habilidades profesionales de quien la recibe. De nada sirve que los cursos estén bien diseñados, brillantemente impartidos, o resulten divertidos y excitantes para quienes los reciben, si lo aprendido no tiene que ver en absoluto con las tareas que el profesional desarrolla o puede desarrollar, o con las habilidades que necesita para ello.

La planta académica de la Escuela Preparatoria 9 valora positivamente la pertinencia de su formación en competencias RIEMS (tabla 1). Según estos datos, al menos un $85 \%$ del profesorado declara estar de acuerdo o muy de acuerdo (en adelante se utilizará 
la expresión individuos que están de acuerdo para designar a ambos grupos) en que las competencias enunciadas en el acuerdo 447 de la SEP (2008) son importantes para garantizar la calidad de la enseñanza que se imparte. No puede saberse, a la luz de estos datos, si la SEP acertó al determinar todas las competencias relevantes para los docentes, pero sí que todas las que propuso son consideradas importantes por la mayoría del profesorado. Además, no hay diferencias importantes en el grado de consenso que se produce. En todos los casos, el grado de aceptación se encuentra entre el $85.8 \%$ y el $89.2 \%$. En otras palabras, en el peor de los casos, la determinación de las competencias docentes por parte de los especialistas que diseñaron la RIEMS cuenta con un $14.2 \%$ de disidentes en la Escuela Preparatoria 9.

En el cuestionario empleado en la investigación se prevé también que cada individuo declare si la ayuda que recibió por parte de las autoridades académicas para su formación fue suficiente. Los resultados también fueron muy positivos (tabla 1). En seis de las ocho competencias contempladas se alcanzó un acuerdo por encima del $80 \%$ y en las otras dos el resultado no estuvo muy lejos. La ayuda en materia de formación fue valorada positivamente por un porcentaje mayor de la muestra en el caso de las competencias que se desarrollan en clase o tienen que ver con la relación con los alumnos; por ejemplo, la habilidad para provocar ambientes de aprendizaje adecuados (87.8\%), la capacidad para evaluar con enfoque formativo (86.1\%), o la capacidad para planificar el aprendizaje por competencias (84.4\%). En el otro extremo, el apoyo formativo que fue declarado suficiente por un menor número de individuos versa sobre actividades que competen exclusivamente al docente o al equipo de docentes: la capacidad del docente para organizar su propia formación a lo largo de su vida profesional $(75.8 \%)$ o las habilidades asociadas a la colaboración con otros docentes en los proyectos de mejora continua de la escuela y con los cuadros directivos en la gestión institucional. En cualquier caso, las diferencias no fueron tan importantes como para que justifiquen un análisis individualizado.

La capacitación en el desarrollo de una determinada competencia es eficaz si dicha competencia es adquirida por el individuo que la recibe. En el caso de las competencias docentes de la RIEMS las certificaciones otorgadas por las instituciones que imparten formación podrían interpretarse como una prueba de la eficacia de la formación; pero distintas razones, incluida la variedad de cursos y acciones formativas que los individuos encuestados han podido seguir, hacen pensar que una autoevaluación por parte de los docentes también es pertinente. Las respuestas del profesorado ofrecen una imagen muy favorable de la adquisición de competencias RIEMS por parte del profesorado de la Escuela 9. Las respuestas afirmativas están en todos los casos por encima del umbral del 83\% y hay algunas competencias que, según los individuos consultados, han sido adquiridas por prácticamente el $90 \%$ de la planta docente de la escuela.

$\mathrm{Ni}$ siquiera se aprecian diferencias significativas según el tipo de competencias: la adquisición de competencias docentes es prácticamente similar para las competencias administrativas (planificación competencial y contextual, evaluación en competencias y gestión institucional) que para las competencias académicas (el resto), de acuerdo con la clasificación propuesta por Castro (2015).

De las respuestas del profesorado sobre la importancia de las competencias contempladas en la RIEMS y la suficiencia de la formación recibida puede elaborarse un indicador que sintetiza una valoración de la formación recibida. La valoración se elabora sobre el supuesto de que se produce un déficit formativo cuando un sujeto considera que una determinada competencia es importante pero, al mismo tiempo, considera que no tiene motivos para declarar que ha recibido formación suficiente para desarrollarla. La tabla 1 propone dos aproximaciones a este planteamiento. Un valor positivo en la columna 
"Déficit formativo (A-B)" refleja que el porcentaje de individuos que entienden que la competencia es importante supera al de los que consideran haber recibido formación suficiente. El déficit formativo es tanto más grave cuanto mayor sea la diferencia. En la columna "Percepción del déficit formativo" se aporta un indicador que ofrece un retrato más fácil y directamente legible. Se trata de una frecuencia condicional: el porcentaje de los individuos encuestados que, habiendo declarado que la competencia es importante, no declararon que la formación recibida en esa competencia fuera suficiente.

\section{Tabla 1. Importancia percibida, formación recibida y posesión de competencias docentes. Déficit formativo}

\begin{tabular}{|c|c|c|c|c|c|}
\hline Competencias RIEMS & $\begin{array}{c}\text { A. Importancia } \\
F_{R A^{3}}\end{array}$ & $\begin{array}{c}\text { B. Ayuda y } \\
\text { formación } \\
\text { suficientes } F R A^{3}\end{array}$ & C. Posesión FRA & $\begin{array}{c}\text { Déficit formativo } \\
A-B\end{array}$ & $\begin{array}{l}\text { Percepción } \\
\text { de déficit } \\
\text { formativo }^{4}\end{array}$ \\
\hline $\begin{array}{l}\text { Organiza su formación continua } \\
\text { a lo largo de su trayectoria } \\
\text { profesional. }\end{array}$ & 87.0 & 75.8 & 87.5 & 11.2 & 16.7 \\
\hline $\begin{array}{l}\text { Participa en los proyectos de } \\
\text { mejora continua de su escuela y } \\
\text { apoya la gestión institucional. }\end{array}$ & 85.8 & 79.0 & 83.0 & 6.8 & 9.5 \\
\hline $\begin{array}{l}\text { Domina y estructura los saberes } \\
\text { para facilitar experiencias de } \\
\text { aprendizaje significativo. }\end{array}$ & 87.0 & 80.9 & 88.0 & 6.1 & 9.3 \\
\hline $\begin{array}{l}\text { Construye ambientes para } \\
\text { el aprendizaje autónomo y } \\
\text { colaborativo. }\end{array}$ & 88.7 & 83.1 & 89.2 & 5.6 & 7.9 \\
\hline $\begin{array}{l}\text { Planifica los procesos de } \\
\text { enseñanza y de aprendizaje } \\
\text { atendiendo al enfoque por } \\
\text { competencias, y los ubica } \\
\text { en contextos disciplinares, } \\
\text { curriculares y sociales amplios. }\end{array}$ & 87.5 & 84.4 & 89.2 & 3.1 & 6.0 \\
\hline $\begin{array}{l}\text { Lleva a la práctica procesos de } \\
\text { enseñanza y de aprendizaje } \\
\text { de manera efectiva, creativa } \\
\text { e innovadora a su contexto } \\
\text { institucional. }\end{array}$ & 86.4 & 84.0 & 86.9 & 2.4 & 6.0 \\
\hline $\begin{array}{l}\text { Evalúa los procesos de } \\
\text { enseñanza y de aprendizaje con } \\
\text { un enfoque formativo. }\end{array}$ & 88.1 & 86.1 & 88.1 & 2.0 & 4.6 \\
\hline $\begin{array}{l}\text { Contribuye a la generación } \\
\text { de un ambiente que facilite el } \\
\text { desarrollo sano e integral de los } \\
\text { estudiantes. }\end{array}$ & 89.2 & 87.8 & 89.2 & 1.4 & 3.2 \\
\hline
\end{tabular}

\footnotetext{
${ }^{3}$ Las columnas FRA recogen la frecuencia relativa de los individuos que declaran estar "de acuerdo" o "muy de acuerdo" con las preguntas sobre la importancia de las competencias docentes y sobre la suficiencia del apoyo recibido por parte de las autoridades académicas para su formación, y su posesión de dichas competencias respectivamente.

${ }^{4}$ De los individuos que declaran que la competencia es importante, porcentaje que representan quienes no declaran haber recibido formación y ayuda suficientes.
} 
El primer indicador del déficit formativo muestra varios hechos. En primer lugar, la diferencia es siempre positiva, es decir, la percepción de importancia está más extendida que la de suficiencia del apoyo para las acciones formativas en todas y cada una de las competencias. En segundo lugar, el déficit es claramente mayor en unas competencias que en otras (en la tabla, las ocho competencias RIEMS se han ordenado precisamente de acuerdo con este criterio). El déficit es mayor en lo que respecta a la capacidad del profesor para organizar su propio aprendizaje y desarrollo profesional; a su capacidad para participar con sus colegas en la mejora continua de la escuela; y a su capacidad para dominar y estructurar saberes con el objeto de diseñar experiencias de aprendizaje significativo. Se aprecia con facilidad que dos de estas competencias tienen que ver con la gestión que deben llevar a cabo maestras y maestros de sus propios conocimientos.

El segundo indicador de déficit formativo muestra, por ejemplo, que en lo que respecta a la competencia de gestión de la formación continua, el $16.7 \%$ de los individuos que consideran que dicha competencia es importante piensa que la formación recibida en este aspecto no es suficiente. El porcentaje no es ni mucho menos mayoritario, pero hace pensar que, en esa competencia en particular, podría realizarse un mayor esfuerzo formativo, o bien en que se debería revisar la eficacia de los procedimientos que se están aplicando. En el resto de las competencias este indicador contribuye a destacar que la percepción mayoritaria es que la formación recibida ha sido suficiente, a pesar de que siempre existen individuos (en cualquier caso menos que uno de cada diez) que consideran que no recibieron formación suficiente en unas competencias que consideran importantes para la calidad de su trabajo.

\section{Prácticas docentes en la RIEMS: importancia atribuida y aplicación por parte del profesorado}

Las reformas educativas sólo son eficaces si provocan un cambio en el comportamiento del personal docente, tanto fuera como, principalmente, dentro del aula. Exceptuando las medidas que tienen que ver con la inversión en recursos materiales (salas de cómputo o instalaciones deportivas, por ejemplo), o personales (contratación de más personal docente), los demás intentos de mejorar la calidad de la enseñanza sólo pueden ser eficaces cuando consiguen influir en la práctica diaria de las maestras y los maestros. De ahí el escepticismo con que los expertos en reformas educativas valoran las reformas que se llevan a cabo al margen del profesorado (Díaz-Barriga, 2010; López y Tinajero, 2009), y que ignoran la necesidad de vencer las inercias que se producen en su actividad. La RIEMS no se limita a describir las virtudes de un enfoque pedagógico desde un punto de vista abstracto e ideal, sino que especifica las competencias que debe poseer cada docente y también los atributos en que se concretan las competencias, algunos de los cuales pueden expresarse como prácticas concretas que se espera que todo el personal docente desarrolle en su trabajo.

Las respuestas que se recabaron en la Escuela 9 del SEMS de la UdeG sugieren que su profesorado se encuentra mayoritariamente de acuerdo con la necesidad de aplicar las prácticas docentes seleccionadas (tabla 2). Sólo una de ellas, la utilización de textos en otros idiomas, no cuenta con el visto bueno de la mayoría de los individuos encuestados. El resto recibe el apoyo de, al menos, el 80\% de la plantilla docente de la escuela, y en la mayoría de los casos este apoyo se eleva hasta prácticamente el $90 \%$ del profesorado.

Se puede concluir, por tanto, que el acuerdo con las prácticas docentes es más o menos equivalente al que reciben las competencias RIEMS, con unas pocas excepciones; lo cual indica que el profesorado apoya el diseño de la RIEMS no sólo en el nivel más abstracto de las competencias que el profesorado debe reunir, sino también en el más concreto, y significante para él, de la renovación de las actividades propias de la profesión de maestro. Se percibe, lo mismo que en el caso de las competencias, tan sólo 
Tabla 2. Importancia percibida, formación recibida y aplicación de competencias docentes. Déficit formativo

\begin{tabular}{|c|c|c|c|c|c|}
\hline Prácticas & $\begin{array}{l}\text { A. Importancia } \\
\text { FRA }^{5}\end{array}$ & $\begin{array}{c}\text { B. Ayuda y } \\
\text { formación } \\
\text { suficientes } F R A^{5}\end{array}$ & $\begin{array}{l}\text { C. Aplicación } \\
\text { FRA }^{5}\end{array}$ & $\begin{array}{l}\text { Déficit } \\
\text { formativo } \\
(A-B)\end{array}$ & $\begin{array}{l}\text { Percepción } \\
\text { de déficit } \\
\text { formativo }^{6}\end{array}$ \\
\hline $\begin{array}{l}\text { Utilización de textos en otros } \\
\text { idiomas. }\end{array}$ & 59.7 & 43.8 & 42.1 & 15.9 & 40.8 \\
\hline Diseño de materiales didácticos. & 89.2 & 75.4 & 83.0 & 13.8 & 17.9 \\
\hline $\begin{array}{l}\text { Diseño de estrategias de } \\
\text { aprendizaje basadas en } \\
\text { proyectos e investigaciones } \\
\text { disciplinarios e } \\
\text { interdisciplinarios. }\end{array}$ & 88.6 & 76.1 & 81.8 & 12.5 & 16.7 \\
\hline $\begin{array}{l}\text { Resolución de conflictos en el } \\
\text { aula. }\end{array}$ & 87.5 & 75.2 & 87.6 & 12.3 & 19.0 \\
\hline $\begin{array}{l}\text { Asesoramiento a los alumnos } \\
\text { sobre el uso de la biblioteca. }\end{array}$ & 83.0 & 71.2 & 83.5 & 11.8 & 17.2 \\
\hline $\begin{array}{l}\text { Seguimiento académico } \\
\text { individualizado de los alumnos. }\end{array}$ & 87.5 & 76.7 & 88.1 & 10.8 & 15.6 \\
\hline Innovación educativa. & 90.3 & 80.1 & 88.1 & 10.2 & 12.6 \\
\hline $\begin{array}{l}\text { Diseño y utilización de } \\
\text { mecanismos de autoevaluación } \\
\text { y evaluación de los alumnos. }\end{array}$ & 90.9 & 81.3 & 88.1 & 9.6 & 11.9 \\
\hline $\begin{array}{l}\text { Motivación del alumnado al } \\
\text { aprendizaje. }\end{array}$ & 89.7 & 82.7 & 92.1 & 7.0 & 10.3 \\
\hline $\begin{array}{l}\text { Evaluación diagnóstica al inicio } \\
\text { del curso. }\end{array}$ & 89.2 & 82.8 & 88.1 & 6.4 & 9.6 \\
\hline $\begin{array}{l}\text { Promoción de ambientes de } \\
\text { aprendizaje. }\end{array}$ & 91.5 & 85.3 & 91.5 & 6.2 & 8.7 \\
\hline $\begin{array}{l}\text { Uso de tecnologías de } \\
\text { información y comunicación en } \\
\text { el aula. }\end{array}$ & 90.3 & 84.6 & 89.7 & 5.7 & 8.9 \\
\hline Planeación colegiada. & 89.8 & 85.8 & 90.9 & 4.0 & 7.0 \\
\hline
\end{tabular}




\begin{tabular}{|l|c|c|c|c|c|}
\hline Autoevaluación. & 88.1 & 84.1 & 87.5 & 4.0 & 9.0 \\
\hline $\begin{array}{l}\text { Enfoque constructivista de la } \\
\text { enseñanza. }\end{array}$ & 89.2 & 85.7 & 90.9 & 3.5 & 3.8 \\
\hline $\begin{array}{l}\text { Evaluación por pares } \\
\text { académicos. }\end{array}$ & 85.8 & 82.8 & 81.2 & 3.0 & 7.3 \\
\hline $\begin{array}{l}\text { Evaluación de los procesos de } \\
\text { aprendizaje con un enfoque } \\
\text { formativo. }\end{array}$ & 89.8 & 87.5 & 91.5 & 5.3 & 5.7 \\
\hline $\begin{array}{l}\text { Comunicación con los alumnos } \\
\text { por medios electrónicos fuera } \\
\text { del aula. }\end{array}$ & 80.2 & 79.5 & 80.1 & 0.7 & 11.3 \\
\hline \begin{tabular}{l} 
Enfoque por competencias. \\
\hline
\end{tabular}
\end{tabular}

en torno a un $10 \%$ de personal escéptico, aunque no necesariamente beligerante, con el diseño de la reforma en cuanto a sus efectos sobre el profesorado.

Sí se perciben diferencias, en cambio, en el modo en que el profesorado valora la formación y la ayuda que ha recibido en el desarrollo de competencias y prácticas docentes. Una vez más la valoración es positiva en este capítulo, es decir, la formación y la ayuda es considerada suficiente por una mayoría del profesorado; sin embargo, existen algunas prácticas en las que esa valoración positiva procede de no mucho más del $75 \%$ del profesorado encuestado. Esto implica que existe en torno a un $25 \%$ del personal que no ha querido declarar que la formación recibida haya sido suficiente en aspectos como la utilización de textos en otros idiomas, el diseño de materiales didácticos, la utilización de metodologías de aprendizaje basadas en proyectos, la resolución de conflictos en el aula, o el asesoramiento de los alumnos en el uso de la biblioteca.
$\mathrm{Al}$ igual que en el análisis de las competencias docentes, se han calculado las diferencias entre las frecuencias relativas de las respuestas de apoyo a la importancia de las prácticas docentes y las de las respuestas que declaran que la formación y la ayuda recibidas han sido suficientes. Y también ocurre esta vez que todas las diferencias son positivas; es decir, que no hay una sola práctica docente en la que ocurra que la percepción de su importancia se encuentre más extendida entre el profesorado que la percepción de que se ha recibido formación suficiente para ejercerla. Se observa, asimismo, que las diferencias no son demasiado abultadas, pero sí de mayor magnitud y más frecuentes que en el caso de las competencias: si había solamente una competencia RIEMS (de ocho) en la que la diferencia superase el $10 \%$, en este caso se pueden localizar siete prácticas, de 19, en las que ocurre otro tanto.

Lo mismo puede decirse cuando se emplea el otro indicador del déficit formativo. Ocurre en 10 (de las

\footnotetext{
${ }^{5}$ Las columnas FRA recogen la frecuencia relativa de los individuos que declaran estar "de acuerdo" o "muy de acuerdo" con las preguntas sobre la importancia de las prácticas docentes, sobre la suficiencia del apoyo recibido por parte de las autoridades académicas para su formación, y sobre su adecuada aplicación, respectivamente.

${ }^{6}$ De los individuos que declaran que la práctica es importante, porcentaje que representan quienes no declaran haber recibido formación y ayuda suficientes.
} 
19) prácticas docentes que más de un $10 \%$ de los individuos que consideran dicha práctica importante no declara haber recibido formación y ayuda suficiente para implementarla. Los casos más destacados son: la utilización de textos en otros idiomas $(41 \%)$; la resolución de conflictos en el aula (19\%); el diseño de materiales didácticos (18\%); el asesoramiento de los alumnos en el uso de la biblioteca (17\%); el diseño de estrategias de aprendizaje basado en proyectos (17\%) o el seguimiento individualizado a los alumnos $(16 \%)$.

En resumen, la valoración general que el profesorado de la Escuela Preparatoria 9 ha realizado de la formación recibida es buena en términos generales; pero también es cierto que los déficits formativos se aprecian más en el terreno, más concreto y cotidiano, de las prácticas docentes que en el de las competencias del profesorado.

\section{La influencia de la participación en PROFORDEMS}

Este apartado tiene como objetivo verificar si la participación de los profesores en PROFORDEMS ejerce alguna influencia en cada una de las tres facetas que se han considerado en los apartados anteriores: importancia, valoración del apoyo recibido de las autoridades, y posesión de las competencias y aplicación de las prácticas docentes recogidas en la RIEMS. Se valora a continuación cómo podría interpretarse la influencia en cada uno de estos sentidos:

- Que PROFORDEMS influya en la importancia otorgada a las competencias puede entenderse como una medida de su eficacia socializadora, podría decirse en tal caso que el programa logra convencer a quienes participan en él de la bondad del diseño de la RIEMS.

- Que PROFORDEMS influya en la percepción de que el apoyo recibido por parte de las autoridades para la formación ha sido suficiente, mide hasta qué punto PROFORDEMS representa una diferencia con respecto a otras acciones formativas en las que los sujetos han participado. Ello estaría indicando que, en una planta docente que se ha visto sometida a diversas acciones formativas, la parte más dispuesta a juzgar que el apoyo ha sido suficiente es la que ha participado en PROFORDEMS.

- Que PROFORDEMS influya en la percepción de posesión de las competencias y de aplicación de las prácticas es un indicador de la eficacia que el profesorado atribuye al programa, ya que la finalidad expresa de éste es precisamente que el personal adquiera dichas competencias y sea capaz de desarrollar dichas prácticas.

La influencia de PROFORDEMS se ha establecido empleando pruebas Chi cuadrado para determinar si los porcentajes de acuerdo con cada afirmación son diferentes en dos grupos de individuos: los que han participado en PROFORDEMS y los que no lo han hecho. Para efectuar las pruebas, las escalas de Likert que miden el acuerdo con las diferentes afirmaciones se han transformado para contemplar tan solo dos categorías: por un lado quienes han declarado estar "de acuerdo" o "muy de acuerdo" con cada afirmación, y por otro quienes han elegido cualquiera de las restantes categorías de respuesta. Los resultados de las pruebas se resumen en las tablas 3 (para las competencias) y 4 (para las prácticas docentes). En ellas se expone el porcentaje de los individuos que se mostraron de acuerdo o muy de acuerdo con cada afirmación en función de si disponen, o no, de PROFORDEMS. También se expone la significatividad de la prueba Chi cuadrado. Se han resaltado con un asterisco las pruebas con valores de significatividad menores de 5\%, esto es, los casos en los que puede afirmarse que la incidencia del acuerdo con las afirmaciones propuestas es diferente entre quienes participaron en PROFORDEMS y quienes no lo hicieron.

$\mathrm{Si}$ se considera en primer lugar la influencia de PROFORDEMS en las respuestas del profesorado 


\begin{tabular}{|c|c|c|c|c|c|c|c|c|c|}
\hline 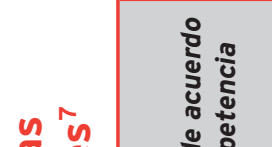 & $\begin{array}{l}\frac{*}{*} \\
\stackrel{*}{0}\end{array}$ & 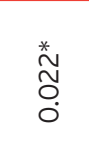 & $\begin{array}{l}* \\
\stackrel{*}{0} \\
\stackrel{0}{0} \\
0\end{array}$ & 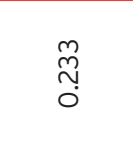 & $\stackrel{\circ}{\circ}$ & $\begin{array}{l}\tilde{n} \\
\stackrel{n}{0} \\
0\end{array}$ & $\begin{array}{l}0 \\
\stackrel{0}{0} \\
0 \\
0\end{array}$ & $\begin{array}{l}\stackrel{\sim}{N} \\
\text { ก } \\
0\end{array}$ & $\begin{array}{l}\stackrel{*}{*} \\
\stackrel{0}{0} \\
\stackrel{0}{0}\end{array}$ \\
\hline 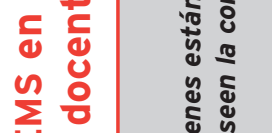 & ¿े & $\begin{array}{l}\circ \\
\infty \\
\infty \\
\circ\end{array}$ & 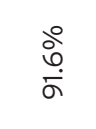 & $\begin{array}{l}\stackrel{0}{0} \\
\infty \\
\stackrel{0}{0}\end{array}$ & $\begin{array}{l}\stackrel{0}{े} \\
\stackrel{\infty}{\infty}\end{array}$ & $\begin{array}{l}\stackrel{0}{0} \\
\infty \\
\text { वें }\end{array}$ & $\begin{array}{l}\text { oे } \\
\text { à }\end{array}$ & $\frac{\circ}{8}$ & $\begin{array}{c}\stackrel{0}{0} \\
\stackrel{0}{\infty}\end{array}$ \\
\hline 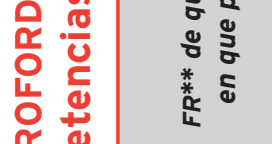 & $\frac{5}{n}$ & $\begin{array}{l}\stackrel{\circ}{\infty} \\
\stackrel{1}{\Gamma}\end{array}$ & $\begin{array}{l}\stackrel{\circ}{\infty} \\
\stackrel{1}{\Gamma}\end{array}$ & $\begin{array}{l}\stackrel{\circ}{+} \\
\stackrel{\infty}{+}\end{array}$ & $\begin{array}{l}\circ \\
\stackrel{0}{0} \\
\infty\end{array}$ & $\begin{array}{l}\circ \\
\stackrel{0}{0} \\
\stackrel{0}{\infty}\end{array}$ & $\underset{\infty}{\stackrel{\sim}{N}}$ & $\begin{array}{l}\stackrel{0}{\hat{0}} \\
\stackrel{0}{\infty}\end{array}$ & $\stackrel{\stackrel{\circ}{i}}{\stackrel{2}{r}}$ \\
\hline 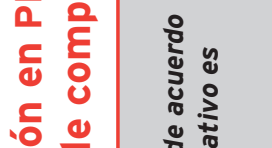 & 菜 & 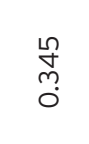 & $\stackrel{\widetilde{m}}{0}$ & $\begin{array}{l}\stackrel{*}{*} \\
\stackrel{5}{0} \\
0\end{array}$ & $\begin{array}{l}\stackrel{\circ}{\%} \\
\text { ஸे }\end{array}$ & $\stackrel{\substack{\infty \\
m}}{0}$ & $\underset{O}{\stackrel{ \pm}{二}}$ & $\begin{array}{l}\stackrel{0}{Y} \\
\stackrel{0}{0}\end{array}$ & $\begin{array}{l}\bar{N} \\
0 \\
0\end{array}$ \\
\hline 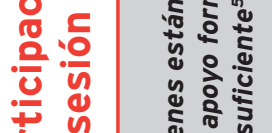 & ذे & $\stackrel{\text { in }}{\stackrel{i}{\wedge}}$ & $\begin{array}{l}\stackrel{0}{8} \\
\ddot{\infty} \\
\infty\end{array}$ & $\begin{array}{l}\stackrel{\circ}{8} \\
\stackrel{\infty}{\infty}\end{array}$ & $\begin{array}{l}\stackrel{\circ}{+} \\
\stackrel{\infty}{\infty}\end{array}$ & & $\begin{array}{l}\circ \\
\infty \\
\omega \\
\infty \\
\infty\end{array}$ & $\begin{array}{l}\stackrel{0}{\circ} \\
\infty \\
\infty \\
\infty\end{array}$ & $\frac{\stackrel{\circ}{\bar{\infty}}}{\overline{\overline{0}}}$ \\
\hline 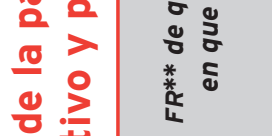 & ติ & $\begin{array}{l}\text { in } \\
\text { in }\end{array}$ & 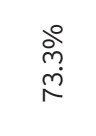 & $\begin{array}{l}\stackrel{\circ}{\circ} \\
\text { م. }\end{array}$ & $\begin{array}{l}\stackrel{0}{0} \\
\dot{0} \\
\infty\end{array}$ & 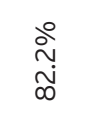 & 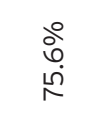 & $\begin{array}{l}\stackrel{+}{+} \\
\stackrel{\infty}{+}\end{array}$ & $\stackrel{\stackrel{\circ}{\rho}}{\stackrel{p}{\Gamma}}$ \\
\hline 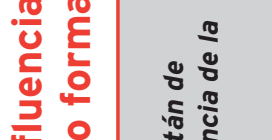 & 悉 & 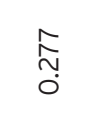 & $\begin{array}{l}\stackrel{*}{n} \\
\stackrel{N}{0} \\
0 \\
0\end{array}$ & $\stackrel{\stackrel{L n}{N}}{\stackrel{0}{0}}$ & $\begin{array}{l}\text { กิ } \\
\text { Oִ } \\
0\end{array}$ & $\frac{\bar{\sigma}}{\circ}$ & $\stackrel{\circ}{=}$ & 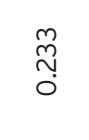 & $\begin{array}{l}\stackrel{0}{\mathcal{Z}} \\
\stackrel{0}{0}\end{array}$ \\
\hline 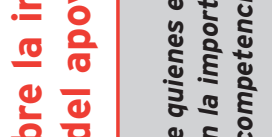 & ذ̊ & $\begin{array}{l}\stackrel{\circ}{\circ} \\
\infty \\
\infty \\
\infty\end{array}$ & $\frac{\circ}{\circ}$ & ळें & $\begin{array}{l}\stackrel{\circ}{\rho} \\
\stackrel{\infty}{\infty}\end{array}$ & $\frac{\circ}{\circ}$ & $\begin{array}{l}\circ \\
\infty \\
\circ \\
\circ\end{array}$ & $\begin{array}{l}\stackrel{0}{\circ} \\
\infty \\
\stackrel{0}{\circ}\end{array}$ & $\begin{array}{l}\stackrel{0}{0} \\
\stackrel{0}{\infty}\end{array}$ \\
\hline 卷这 & ติ & $\underset{\infty}{\stackrel{\circ}{N}}$ & $\begin{array}{l}\stackrel{\circ}{\circ} \\
\stackrel{0}{\wedge}\end{array}$ & $\underset{\infty}{\stackrel{\circ}{i ் ~}}$ & $\stackrel{\stackrel{\circ}{\infty}}{\stackrel{\sim}{\wedge}}$ & 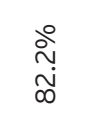 & $\underset{\infty}{\stackrel{\infty}{i ் ~}}$ & $\begin{array}{l}\stackrel{\circ}{\circ} \\
\dot{\infty} \\
\dot{\infty}\end{array}$ & $\underset{\infty}{\stackrel{\sim}{0}}$ \\
\hline \multicolumn{2}{|c|}{ 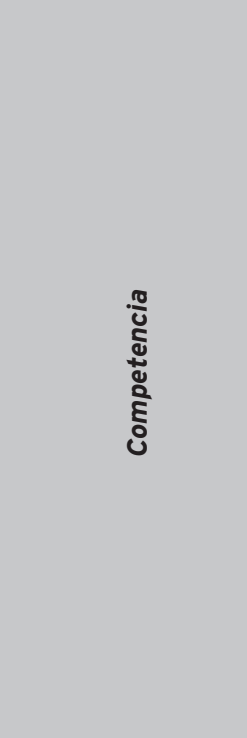 } & 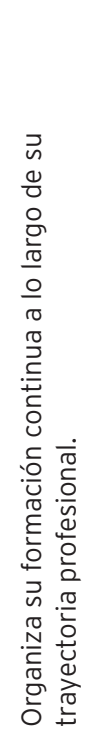 & 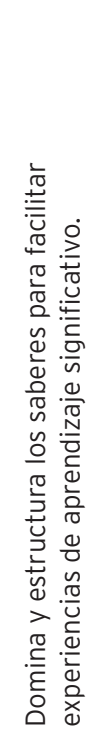 & 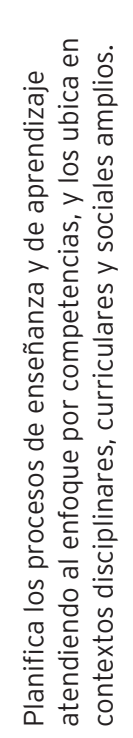 & 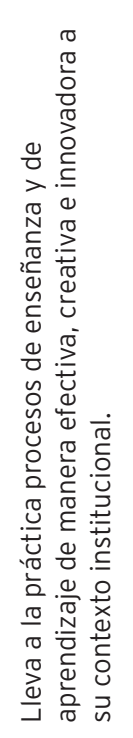 & 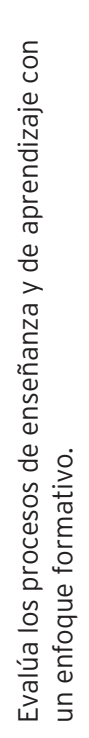 & 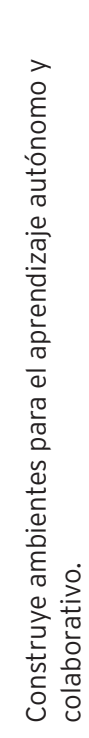 & 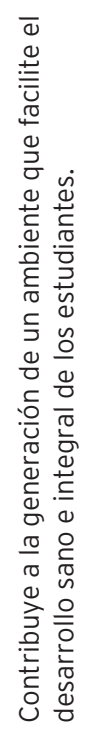 & 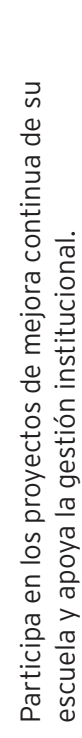 \\
\hline
\end{tabular}




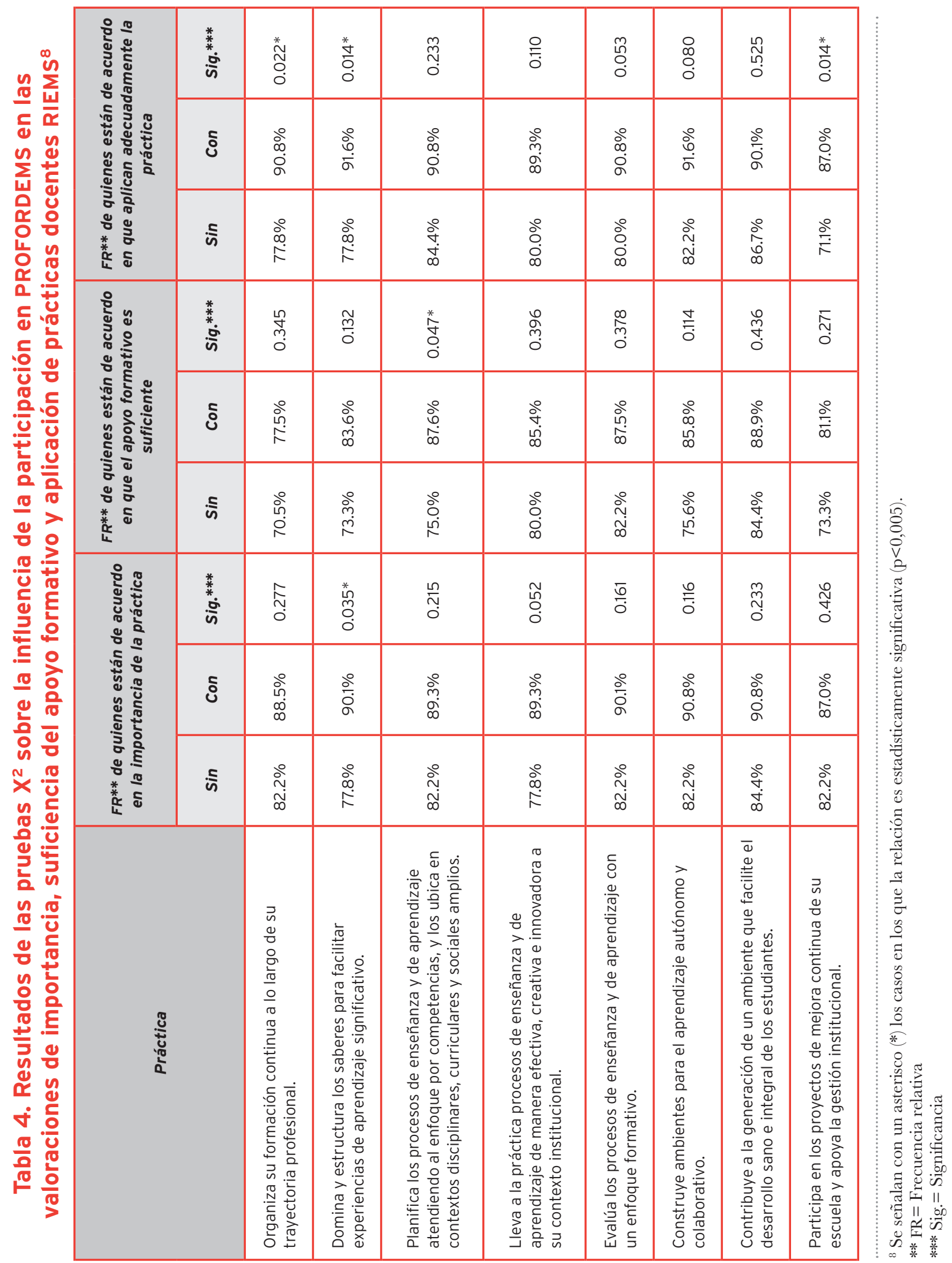


relativas a las competencias docentes, se concluye que PROFORDEMS no ha tenido un efecto socializador muy notable (cf. Castro, 2015); el efecto de la participación en PROFORDEMS sólo es estadísticamente significativo en una de las ocho competencias RIEMS, la que consiste en el dominio de los saberes para facilitar experiencias de aprendizaje significativo. Este resultado no implica que el profesorado en la escuela esté convencido de que la propuesta de competencias docentes en RIEMS resulta incorrecta, ni que considere que su extensión en el profesorado no tiene ningún efecto positivo. Por el contrario, la mayoría del profesorado parece estar de acuerdo con la elección de todas las competencias docentes en la RIEMS; lo que ocurre es que ese convencimiento lo ha adquirido con independencia de su participación en el PROFORDEMS; es decir, probablemente lo ha adquirido con anterioridad, durante su capacitación en la enseñanza por competencias previa a la entrada en vigor de la RIEMS.

Por otro lado, tampoco se percibe influencia de PROFORDEMS en la valoración que el profesorado realiza del apoyo recibido por parte de las autoridades. También en esta ocasión la influencia se aprecia solamente en una de las competencias RIEMS: la competencia que consiste en planificar los procesos de enseñanza y de aprendizaje empleando un enfoque por competencias.

En cambio, el efecto RIEMS es más visible en lo relativo a la eficacia de la formación, o al menos en la percepción que tienen los individuos encuestados de dicha eficacia. Participar en PRFORDEMS incrementa la probabilidad de que un individuo dado considere que está en disposición de tres competencias RIEMS: la organización de su propia formación continua, el manejo de sus conocimientos para generar experiencias de aprendizaje significativo, y su capacidad para colaborar en procesos de mejora institucional.

No es ni necesario ni correcto exagerar la influencia de RIEMS en este sentido. La participación en
PROFORDEMS no determina la percepción subjetiva de posesión de ninguna de las competencias RIEMS. De hecho, la mayoría del personal consultado estima que posee las competencias marcadas por RIEMS, haya participado o no en PROFORDEMS. Por otra parte el efecto se percibe en menos de la mitad de las competencias consideradas. No obstante, se puede decir que la participación en PROFORDEMS influye más en la percepción subjetiva de posesión de las competencias que en la socialización del profesorado en los principios de la RIEMS o en su valoración del papel de las autoridades.

Cuando se considera el efecto de PROFORDEMS en las respuestas a las preguntas sobre prácticas docentes las conclusiones no son radicalmente diferentes, pero sí incorporan algún matiz. Una vez más el efecto de la participación en PROFORDEMS se aprecia mejor en la valoración que realiza el profesorado del apoyo prestado por las autoridades y en la percepción de eficacia de la formación, es decir de la aplicación de las prácticas docentes por parte del profesorado. En estas dos facetas, PROFORDEMS representa una diferencia estadísticamente significativa en cuatro y tres ocasiones respectivamente. En lo que se refiere a la socialización del profesorado en los principios RIEMS, PROFORDEMS sólo ha supuesto diferencias significativas en una de las prácticas docentes, la utilización de textos en otros idiomas. En resumen, tampoco en el caso de las prácticas docentes puede decirse que la participación en PROFORDEMS resulte determinante. La participación en el programa no altera la opinión del profesorado con respecto a la mayoría de las prácticas docentes, ni en lo que se refiere a la importancia que les atribuye, ni en lo relativo a la valoración del apoyo recibido, ni en la faceta quizá más importante que consiste en la adopción efectiva de dichas prácticas.

\section{Conclusiones}

Este artículo se ha realizado con un doble objetivo. Por un lado pretende una evaluación de la 
capacitación recibida por el profesorado de la EMS en lo relativo a las competencias y las prácticas docentes que requiere la RIEMS. Una evaluación de este tipo queda sobradamente justificada por el papel que la formación del profesorado juega en la correcta implantación de la RIEMS, por las opiniones vertidas por diversos expertos con respecto a la escasa capacitación del profesorado de este nivel educativo; y por las reiteradas ocasiones en que dichos expertos han alertado de la necesidad de socializar a los individuos que componen el profesorado de la EMS en los principios que sostienen cualquier reforma.

La evaluación de la reforma que contiene este artículo tiene la particularidad de que se basa en las opiniones de las maestras y maestros. Estas opiniones, como cualesquiera otras, no reflejan necesariamente la realidad de los hechos y pueden estar influidas por factores diversos e independientes de las consecuencias materiales de la propia reforma (Olaskoaga-Larrauri et al., 2017); pero, con todo, representan un interesante contrapunto a la versión oficial sobre los efectos de las reformas o la eficacia de los programas formativos, o a la doctrina de los expertos sobre esta misma materia.

De hecho hay un aspecto en el que las opiniones de maestras y maestros reflejan mejor que ninguna otra las consecuencias de los programas formativos: el de su socialización en los ideales y objetivos de la propia reforma. Si uno de los objetivos de la formación consistía en provocar la adhesión de los docentes a los principios de la enseñanza por competencias y a las medidas adoptadas por la propia reforma, este objetivo sólo puede verificarse recurriendo al universo simbólico de maestras y maestros.

El segundo objetivo del artículo consiste en valorar si la participación en PROFORDEMS provoca diferencias en la valoración que las maestras y los maestros han realizado de la formación recibida. Recuérdese, en este sentido, que la formación del profesorado en competencias y prácticas docentes no se ha limitado a la obtenida a través de
PROFORDEMS; y, en particular, que el profesorado de la Escuela Preparatoria 9, cuyas impresiones se han recogido en este trabajo, ha tenido la oportunidad de participar en numerosas acciones formativas desarrolladas al calor de los planes promovidos por la Universidad de Guadalajara para la puesta en marcha de un bachillerato por competencias en todo su Sistema de Educación Media Superior.

Los resultados obtenidos en el trabajo de campo han sido en cierto modo sorprendentes. La valoración del profesorado consultado ha sido mayoritariamente positiva en las tres facetas consideradas: la influencia prevista de las competencias y las practicas docentes en la calidad educativa; el apoyo recibido por las autoridades académicas en materia formativa; y la asimilación por parte del profesorado tanto de las competencias como de las prácticas docentes.

Es cierto que para algunas profesoras y profesores existen déficits formativos en determinadas competencias y prácticas docentes; no obstante, los resultados en general han sido buenos y reflejan una situación que, aunque mejorable, poco tiene que ver con el tono pesimista que diversos analistas emplearon en sus manifestaciones con respecto a la eficacia de la RIEMS y, en concreto, con respecto a su capacidad para hacer comulgar al personal docente con la reforma y modificar efectivamente su forma de trabajar.

Por otro lado, el PROFORDEMS, declarado "piedra angular de la RIEMS" (López y Tinajero, 2009: 1231), tiene poco que ver, a tenor de la evidencia reunida en este artículo, con las favorables impresiones del profesorado de la Escuela Preparatoria 9. La participación en este programa apenas explica unas pocas diferencias en la opinión del profesorado consultado. Lo cual remite necesariamente a la intensa labor formativa que se llevó a cabo en el SEMS de la UdeG con anterioridad a la reforma.

Desde el punto de vista del diseño de la RIEMS y de la implementación de la reforma, en particular en lo relativo a la formación del profesorado, los 
resultados de este artículo permiten extraer algunas interesantes consecuencias. En primer lugar, no hay razones para pensar en una oposición inevitable a los cambios que propone la RIEMS por parte del profesorado. Las respuestas de las maestras y maestros que componen la plantilla de la Escuela Preparatoria 9 hacen pensar que han asimilado bastante bien los objetivos de la reforma y se han involucrado con ellos, aceptando la nueva manera de practicar la docencia, así como la necesidad de fortalecer las competencias que demanda el nuevo enfoque pedagógico.

En segundo lugar, los resultados no restan importancia a los esfuerzos que la SEP pueda llevar a cabo en el ámbito de la formación del profesorado. La mayor parte de la plantilla de la Escuela 9 ha participado intensamente en acciones formativas y es muy probable que ello se deje notar en sus respuestas con respecto a la aceptación del enfoque por competencias, a la posesión de competencias y la aplicación de las prácticas docentes. En otras palabras, la evidencia que se ha reunido no sirve para sostener que la formación del personal docente sea ineficaz sino, en todo caso, lo contrario.

En tercer lugar, la formación de PROFORDEMS resulta insuficiente, como han puesto de relieve diversos autores, cuando desde las instituciones y los planteles no se diseñan instrumentos de diagnóstico de la situación formativa y de motivación, ayuda y seguimiento al profesorado que participa en el programa. En contra de lo que suele ser habitual, en el SEMS, y más en particular en la Preparatoria 9, esos instrumentos estuvieron presentes desde el inicio de la reforma porque existía una voluntad institucional y compartida de conseguir la incorporación del plantel al Sistema Nacional de Bachillerato. Como resultado, la participación del profesorado en el programa no sólo fue más numerosa, sino también - a juicio de las y los docentes - más eficaz. 


\section{Referencias}

Alcántara, Armando y Juan Fidel Zorrilla (2010), "Globalización y educación media superior en México", en Perfiles Educativos, núm. 32, pp. 38-57.

Auditoría Superior de la Federación (ASF) (2011), Auditoría de desempeño: 11-0-11100-07-0399 [Formación de docentes de la educación media superior], México, Secretaría de Educación Pública (SEP).

Beerkens, Eric (2008), "The emergence and institutionalisation of the European higher education and research area" en European Fournal of Education, núm. 43 (4), pp. 407-425.

Castro Vallés, Alberto (2015), “Competencias administrativas y académicas en el profesorado de educación superior", en Revista Mexicana de Investigación Educativa, núm. 20 (64), pp. 263-294.

Day, Christopher (2002), "School reform and transitions in teacher professionalism and identity", en International Fournal of Educational Research, núm. 37, pp. 677-692.

Díaz-Barriga Arceo, Frida (2010), "Los profesores ante las innovaciones curriculares", en Revista Iberoamericana de Educación Superior, núm. 1 (1), pp. 37-57.

Fonseca, César (2011), "Reforma integral de la educación media superior", en Inventio, la génesis de la cultura universitaria en Morelos, núm. 13, pp. 44-52.

González, Ma. Inez, Claudia Camacho y María del Roble García (2015), "La Reforma Integral de la Educación Media Superior: una mirada desde el asesor virtual", en Revista Mexicana del Bachillerato a Distancia, núm. 7 (14), pp. 59-68.

Haro del Real, Ma. Jesús, José Trinidad Padilla, Lourdes Elizabeth Parga y José Alfredo Peña (2007), Plan de Estudios del Bachillerato General por Competencias en el Sistema de Educación Media Superior. Una propuesta. Tomo I, Guadalajara, México, SEMS de la UdeG.

Ibarra, Luz Marina, Ana Esther Escalante y Ciésar Darío Fonseca (2012), "Docentes del Bachillerato en la Reforma Integral: sus valores profesionales", en IX Fornada Nacional de Investigadores en Educación y Valores, Guadalajara, septiembre, <http://www.
academia.edu/13855870/Docentes_de_bachillerato_ en_la_riems:sus_valores_profesionales $>$ [Consulta: diciembre de 2016].

Imbernón, Francisco (1994), La formación y el desarrollo profesional del profesorado: hacia una nueva cultura profesional, Barcelona, Graó.

Little, Judith Warren (1993), “Teachers' professional development in a climate of educational reform", en Educational Evaluation and Policy Analysis, núm. 15 (2), pp. 129-151.

López Bonilla, Guadalupe y Guadalupe Tinajero Villavicencio (2009), "Los docentes ante la reforma del bachillerato", en Revista Mexicana de Investigación Educativa, núm. 14 (43), pp. 1191-1218.

López, Carmen Minerva (2011), "Evaluación y propuesta para la mejora de la implementación de la Reforma Integral de Educación Media Superior en el Colegio de Bachilleres del Estado de Sonora a partir de la percepción de los docentes", Tesis de Maestría, <http://registromodeloeducativo.sep.gob.mx/Archiv o;jsessionid=3bbfd4717ca4c4471ceb56f9d69b?nomb re $=971-$ Foro + de + consulta + modelo + educativo.doc. $>$ [Consulta: noviembre de 2015].

Lozano Medina, Andrés (2012), "Límites de la reforma en educación media superior", en Perfiles educativos, núm. 34, pp. 164-169.

Lozano Medina, Andrés (2015), "La RIEMS y la formación de los docentes de la educación media superior en México: antecedentes y resultados iniciales", en Perfiles Educativos, núm. 37, pp. 108-124.

Macías Narro, Alfredo (2009), "La RIEMS, un fracaso anunciado", en Odiseo, Revista Electrónica de Pedadogía, núm. 6 (12), pp. 1-43.

Martínez Carazo, Piedad Cristina (2006), "El método de estudio de caso: estrategia metodológica de la investigación científica”, en Pensamiento \& Gestión, núm. 20, pp. 165-193.

Olaskoaga-Larrauri, Jon, Xabier González-Laskibar, Elia Marúm-Espinosa y Eneritz Onaindia- 
Gerrikabeitia (2015), "Reformas organizativas en las IES, condiciones laborales y reacciones de los académicos", en Revista Iberoamericana de Educación Superior, núm. 6 (17), pp. 102-118.

Olaskoaga-Larrauri, Jon, Carlos Mendoza-Sepúlveda y Elia Marúm-Espinosa (2017), “Concepciones sobre calidad educativa en el profesorado del sistema de educación media superior de la Universidad de Guadalajara", en Education Policy Analysis Archives, núm. 25 (81), pp. 1-24.

Scheel Martín, Carlos Guillermo (2009), "Reflexiones en torno a la RIEMS", en Odiseo. Revista Electrónica de Pedagogía, núm. 6 (12).

Schmidt, Michèle y Amanda Datnow (2005), "Teachers' sense-making about comprehensive school reform: the influence of emotions", en Teaching and Teacher Education, núm. 21, pp. 949-965.

SEMS de la UdeG (2009), Informe de Actividades SEMS 2009. Reporte Técnico, Guadalajara, México, SEMS de la UdeG.

SEMS de la UdeG (2008), Informe de Actividades SEMS 2008. Reporte Técnico, Guadalajara, México, SEMS de la UdeG.

Secretaría de Educación Pública (SEP) (2014), Segundo Informe de Labores 2013-2014, México, SEP, <http:// www.planeacion.sep.gob.mx/Doc/informes/ labores/2012_2018/2do_informe_de_labores.pdf.> [Consulta: noviembre de 2015].

SEP (2008), "Acuerdo 447, por el que se establecen las competencias docentes para quienes impartan educación media superior en la modalidad escolarizada", en Diario Oficial de la Federación, Tercera Sección 1, miércoles 29 de octubre.

SEP-SEMS (2013). "Avances y resultados en la educación media superior", XLIV Reunión Ordinaria del Consejo Nacional de Autoridades Educativas, <http://www.ceppemsoax.com/ index.php?option $=$ com_content\&view $=$ article\&id $=7 \&$ It emid=74.> [Consulta: noviembre de 2015].

SEP-SEMS (2009), "Certifican a personal docente del Sistema Nacional de Bachillerato", <http://www.sems. gob.mx/es_mx/sems/certifican_a_personal_docente_ del_sistema_nacional> [Consulta: noviembre de 2015].

Sosa Guerrero, Leticia, y Carlos Miguel Ribeiro (2014), "La formación del profesorado de matemáticas de nivel medios superior en México: una necesidad para la profesionalización docente", en Revista Iberoamericana y de Producción Academica y Gestión Educativa, núm. 1 (1), pp. 1-15.

Van der Berg, R. (2002), “Teacher's meanings regarding educational practice", en Review of Educational Research, núm. 72 (4), pp. 577-625.

Villa Lever, Lorenza (2012), “Tres problemas prioritarios que urge resolver en la educación media superior", en Perfiles Educativos, Entrevistas, núm. 34, pp. 170-175.

Villarreal Larrinaga, Óscar y Jon Landeta Rodríguez (2010), "El estudio de casos como metodología de investigación científica en dirección y economía de la empresa. Una aplicación a la internacionalización", en Investigaciones Europeas de Dirección y Economía de la Empresa, núm. 16 (3), pp. 31-52.

\section{Cómo citar este artículo:}

Olaskoaga-Larrauri, Jon, Carlos Mendoza-Sepúlveda y Elia Marúm-Espinosa (2018), “La formación del profesorado en la RIEMS. Un estudio de caso en la Escuela Preparatoria 9 del SEMS de la Universidad de Guadalajara", en Revista Iberoamericana de Educación Superior (RIES), México, UNAM-IISUE/Universia, vol. IX, Núm. 26, pp. 22-41, [consulta: fecha de última consulta]. 